\title{
RED AND INFRARED PHOTOMETRY OF Be STARS
}

G. C. Kilambi, P. Vivekananda Rao, and M. B. K. Sarma Osmania University, Hyderabad, India

Observations and Reduction

All programme stars including standard stars were observed with the standard RI filters of the Johnson system with a 20-arcsec diaphragm for 4 nights during the 1982-83 observing season. A photoelectric photometer attached to the 1.2 meter reflecting telescope of the Japal-Rangapur Observatory was used for these observations. A dry-ice cooled EMI 9558B photomultiplier tube was used as the detector and the photocurrent was recorded by means of a GR $1230 \mathrm{~A}$ DC-amplifier and a Honeywell Brown chart recorder.

The extinction coefficients for $R$ and (R-I) were determined from the observations of standard stars taken from the list of Johnson (1966) and also from the observations of an early and a late spectral type star on each night and all the observations were corrected for atmospheric extinction. The instrumental magnitudes of a programme star were transformed to the standard system through normal procedures. The mean internal probable error of a single measurement in $R$ and $(R-I)$ is \pm 0.01 and \pm 0.02 respectively.

\section{Analysis}

The intrinsic colors, $(V-R)$ and $(V-I)$, for each star were derived from the value of color excess, $E(B-V)$, given by Schmidt-Kaler (1964) and through standard relations given by Johnson (1965). The apparent visual magnitude, $V$, for each star was taken from the observations of Johnson (1966) and Feinstein (1975). Fig. 1 shows the relation of $(V-R)$ and $(V-I)$ for all programme stars. Except for stars HR 985, HR 1508, $\mathrm{AR} 2148, \mathrm{HR}^{\circ} 2538$ and HR 5193, the indices of all other stars, even though they exhibit some scatter, agrees more or less with that of the standard intrinsic relation obtained by Johnson (1965) for luminosity class $V$. It is well known that for emission-line stars, the determination of the interstellar extinction correction from $E(B-V)$ is not very accurate because of their intrinsic reddening in the visible region. The fact that the indices of the majority of the stars agree with that of the standard relation for luminosity class $V$ implies that the intrinsic reddening in many of these stars is negligible. The variable nature or free-free emission from an ionized envelope or thermal re-emission by circumstellar dust could be the cause of the observed infrared excess in some of the stars that deviate from the normal relationship. It is very difficult to choose any one of the above without further detailed information.

Fig. 2a, b show the relationship between $M_{v}$ and $(V-R)$ and $M$ and (V-I) for all programme stars. The absolute magnitude, $M$, is est ${ }^{\circ}$ mated from the spectral type using the standard relation given by FitzGerald (1970). 
Many of the programme stars appear to lie closer to the standard mainsequence with some scatter in the $\left[M_{v}-(V-R)\right]$ plane, whereas the same stars show considerable scatter in the $\left.[\mathrm{M},-9 \mathrm{~V}-\mathrm{I})_{0}\right]$ plane. In addition, there are a few stars which lie to the left and to the extreme right of the standard main sequence relation in both these diagrams. HR 985, HR 1508, and HR 5193 lie to the left of the normal main sequence relation in both these diagrams. The observed position of these stars on these diagrams suggest that they are emitting considerably less radiation through $R$ and $I$ passbands than in the $V$ passband, compared to their counterparts. A possible explanation for this behaviour could be the presence of thick shells around them or that the opacity of the material within these shells is much higher or that the shells themselves are much cooler in nature than that of the normal Be stars. Additionally, the observed position of HR 2148 and HR 2538 suggest that these two stars are emitting more flux in both $R$ and $I$ passbands than in the $V$ passband. This may be due to thermal re-emission by circumstellar dust but can only be substantiated through far-infrared observations.

Fig. 3a, $b$ show the relation between $M$ and $(V-R)$ and $M$ and $(V-I)$ for all stars observed by us and that of Schmidt-Kaler (1964). The absolute magnitude was estimated from a statistical relation obtained by SchmidtKaler through the observed intrinsic (U-B). Once again, similar peculiarities to those described above can be noticed on these diagrams also. In addition, one interesting point to note is that the absolute magnitudes derived through (U-B) show that these stars are much more luminous than that of the values derived from the spectral type relation. This could be due to the uncertainties associated with the calibration of the statistical relation such as the estimation of the interstellar reddening, intrinsic reddening and also the dispersion caused as a consequence of viewing the rapidly rotating stars under different angles between the line of sight and the axis of rotation. In addition, the estimated intrinsic (U-B) colours of these stars are too blue and are affected by the influence of the second Balmer jump in emission in the ' $U$ ' magnitudes (Divan et a1. 1982).

Finally, HR 1508, HR 1789, HR 1910, HR 2538, HR 2996, HR 3034, HR 3946 , HR 5193 and HD 45677 have shown considerable brightness fluctations in both the $R$ and $I$ passbands when compared to earlier observational data. A detailed version of this analysis will be published later.

\section{References}

Divan, L., Zorec, J. and Briot, D. (1982). IAU Symp. No. 98, 53. Feinstein, A. (1975). PASP 87, 603.

FitzGerald, M. P. (1970). Astro. \& Astrophys. 4, 234. Johnson, H. L. (1965). Astrophys. J. 141, 923.

Johnson, H. L. (1966). Comm. Lunar \& Planetary Labs. 4, 99. Schmidt-Kaler, T. (1964). Veröff. Astro. Inst. Univ. Bonn, No. 70. 
1

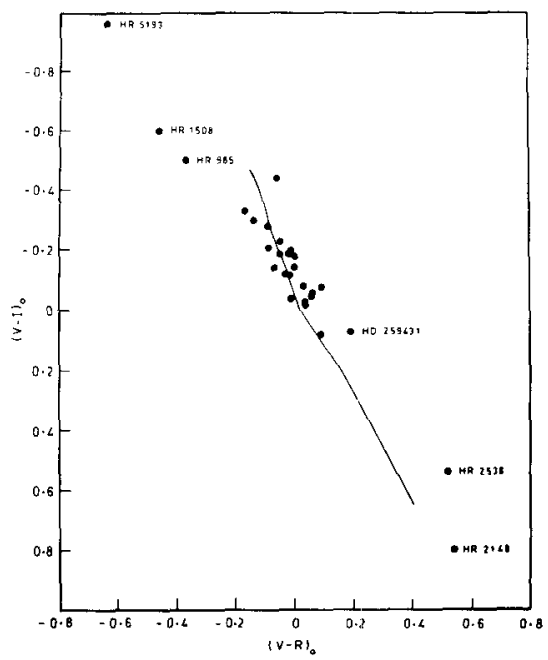

$2 \mathrm{a}$

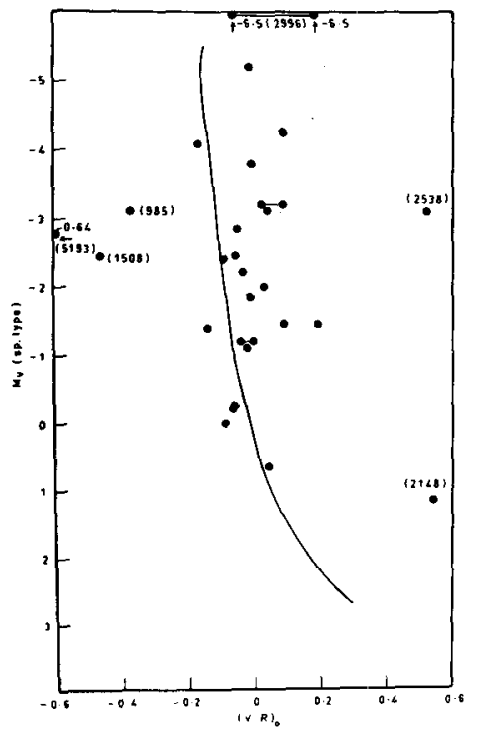

$3 a$

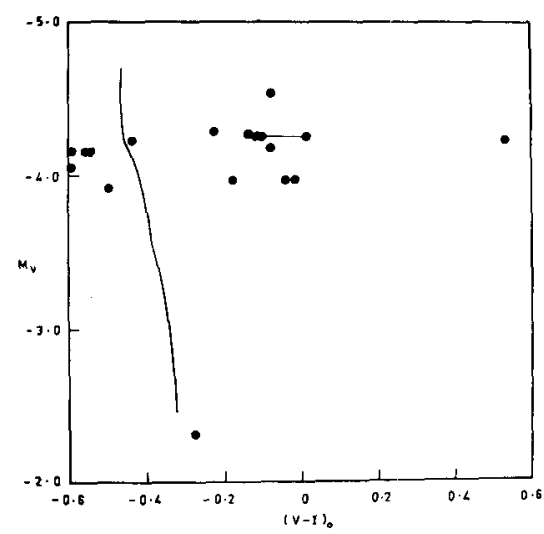

$2 b$

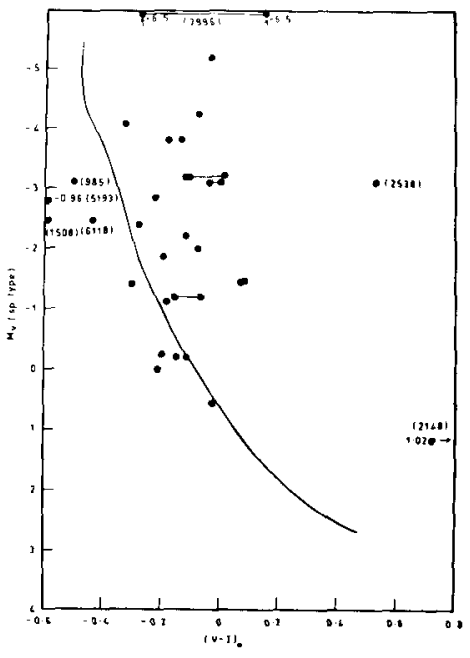

$3 \mathrm{~b}$

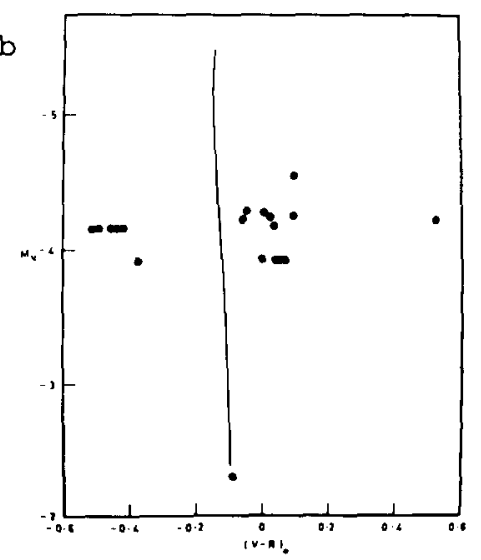




\section{DISCUSSION FOLLOWING KILAMBI}

Peters:

The star HR $2148(=17$ Lep), which stands out in your plot as having a large IR excess, is a mass transfer binary Be star with an $M$ giant companion which, according to IRAS spectral data (LRS), also has dust associated with it (a $10 \mu$ emission peak is observed).

Kilambi:

Probably the infrared excess observed could be due to the dust envelope. More observations in the far-infrared are necessary to verify it.

Polidan:

Regarding the V-I color of HR 2148 (17 Lep, SS Lep), spectroscopic evidence shows that the spectrum of this binary is dominated by the M1III component longward of 7000 A. This would indeed produce a significant near-infrared excess if one is assuming that the near-IR continuum is produced only by the B9 star seen in the optical region.

Kilambi:

I quite agree.

Waters:

The $10 \mu$ feature in the IRAS spectrum of 17 Lep is most likely associated with dust around the M-type giant in this binary system. Such dust emission is quite common in M-type giants and is probably unrelated to the Be star in the system.

Kilambi:

Quite possible. But one has to check whether the near infrared excess observed is wholly from the M-giant only or from the Be star envelope itself or a combination of both. 